\title{
Kidney stones and kidney function loss: a cohort study
} (@) $\mathbb{Q} \Theta$ OPEN ACCESS

\author{
$\mathrm{R}$ Todd Alexander assistant professor ${ }^{1}$, Brenda $\mathrm{R}$ Hemmelgarn associate professor $^{2}$, Natasha \\ Wiebe research associate ${ }^{1}$, Aminu Bello research fellow ${ }^{1}$, Catherine Morgan assistant professor ${ }^{1}$, \\ Susan Samuel assistant professor ${ }^{2}$, Scott W Klarenbach associate professor ${ }^{1}$, Gary C Curhan \\ professor $^{3}$, Marcello Tonelli professor ${ }^{1}$, for the Alberta Kidney Disease Network
}

${ }^{17}$ 7-129 Clinical Science Building, University of Alberta, 8440112 Street, Edmonton, Alberta T6B 2G3, Canada; ${ }^{2}$ University of Calgary, Calgary, Canada; ${ }^{3}$ Channing Laboratory, Harvard University, Boston, USA

\begin{abstract}
Objective To investigate whether the presence of kidney stones increase the risk of end stage renal disease (ESRD) or other adverse renal outcomes.

Design A registry cohort study using validated algorithms based on claims and facility utilisation data. Median follow-up of 11 years.

Setting Alberta, Canada, between 1997 and 2009.

Participants 3089194 adult patients without ESRD at baseline or a history of pyelonephritis. Of these, 1954836 had outpatient serum creatinine measurements and were included in analyses of chronic kidney disease and doubling of serum creatinine level.
\end{abstract}

Exposure One or more kidney stones during follow-up.

Main outcome measures Incident ESRD, development of stage 3b-5 chronic kidney disease (estimated glomerular filtration rate $<45$ $\mathrm{mL} / \mathrm{min} / 1.73 \mathrm{~m}^{2}$ ), and sustained doubling of serum creatinine concentration from baseline.

Results 23706 (0.8\%) patients had at least one kidney stone, 5333 (0.2\%) developed ESRD, 68525 (4\%) developed stage 3b-5 chronic kidney disease, and 6581 (0.3\%) experienced sustained doubling of serum creatinine. Overall, one or more stone episodes during follow-up was associated with increased risk of ESRD (adjusted hazard ratio 2.16 (95\% Cl 1.79 to 2.62$)$ ), new stage 3b-5 chronic kidney disease (hazard ratio 1.74 (1.61 to 1.88$)$ ), and doubling of serum creatinine (hazard ratio 1.94 (1.56 to 2.43$)$ ), all compared with those without kidney stones during follow-up. The excess risk of adverse outcomes associated with at least one episode of stones seemed greater in women than in men, and in people aged $<50$ years than in those aged $\geq 50$. However, the risks of all three adverse outcomes in those with at least one episode of stones were significantly higher than in those without stones in both sexes and age strata. The absolute increase in the rate of adverse renal outcomes associated with stones was small: the unadjusted rate of ESRD was 2.48 per million person days in people with one or more episodes of stones versus 0.52 per million person days in people without stones.
Conclusion Even a single kidney stone episode during follow-up was associated with a significant increase in the likelihood of adverse renal outcomes including ESRD. However, the increases were small in absolute terms.

\section{Introduction}

End stage renal disease (ESRD), chronic kidney disease, and kidney stones are common clinical problems with considerable socioeconomic impact. ${ }^{1-3}$ Patients with rare genetic disorders that cause nephrolithiasis (such as primary hyperoxaluria and cystinuria) may develop ESRD as a consequence of their stone disease. ${ }^{4-8}$ However, kidney stones are infrequently implicated as the primary cause of ESRD (0.2-3.2\% of all ESRD cases). ${ }^{5} 910$ Limited evidence suggests an association between kidney stones and the development of chronic kidney disease. ${ }^{11-14}$ However, whether kidney stones are a risk factor for the development of ESRD is unclear. ${ }^{13}$

Because kidney stones are common and potentially preventable, an accurate assessment of their potential long term consequences would be important to inform health interventions. We used a large, population based cohort of people receiving care in a universal healthcare system in Canada to study this issue. We hypothesised that an episode of kidney stones would be associated with excess risk of adverse renal outcomes, including ESRD, development of clinically relevant chronic kidney disease (stage $3 \mathrm{~b}-5$; defined by estimated glomerular filtration rate $<45$ $\mathrm{mL} / \mathrm{min} / 1.73 \mathrm{~m}^{2}$ ) or sustained doubling of serum creatinine concentration from baseline.

\section{Methods}

\section{Population and data sources}

This study used the Alberta Kidney Disease Network database, which incorporates data from Alberta Health and Wellness (the provincial health ministry), the Northern and Southern Alberta 
Renal Programs, and the clinical laboratories in Alberta. ${ }^{15}$ The database was used to assemble a cohort of adults aged $\geq 18$ years who resided in Alberta, Canada, between April 1997 and March 2009. All people registered with Alberta Health and Wellness were eligible for inclusion. All Alberta residents are eligible for insurance coverage by Alberta Health and Wellness, and $>99 \%$ participate in this coverage. We excluded patients with ESRD (defined as documented estimated glomerular filtration rate $<15$ $\mathrm{mL} / \mathrm{min} / 1.73 \mathrm{~m}^{2}$, chronic dialysis, or prior kidney transplant) at baseline. Because of the known relation between pyelonephritis and kidney stones (and the potential for pyelonephritis to cause kidney failure), we excluded patients with pyelonephritis before or during follow-up. To identify pyelonephritis we used the ICD-9 (international classification of diseases, ninth revision) codes 590, 590.10, 590.00, 590.80, 590.2, 590.9, 590.80, and 590.3 and ICD-10 codes N10, N11.0, N11.1, N11.8, N11.9, N12, N13.6, N15.1, N15.9, N16.0, $\mathrm{N} 28.83$, N28.84, and N28.85. We followed patients from April 1997, their 18th birthday, or registration with Alberta Health and Wellness (whichever was later) until March 2009.

\section{Presentation with kidney stone episodes}

We used physician claims, data on use of hospitalisation and ambulatory care, and ICD-9 codes $(592,594,274.11)$ and ICD-10 codes (N20.0, N20.1, N20.2, N20.9, N21.0, N21.1, $\mathrm{N} 21.8, \mathrm{~N} 21.9, \mathrm{~N} 22.0, \mathrm{~N} 22.8)$ to identify presentations of kidney stones. The accuracy of these codes in defining a kidney stone episode has been validated. ${ }^{12}$ One or more of these codes in any position for any inpatient or outpatient claim was taken to represent an episode of kidney stones. We assumed that an interval of a year or more between claims represented separate kidney stone episodes; claims occurring within a year of each other were classified as a result of a single stone episode. ${ }^{16}{ }^{17}$

\section{Other covariates}

Demographic variables included age (categorised as 18-49, 50-69, and $\geq 70$ years), sex, Aboriginal (registered First Nations or recognised Inuit), receipt of social assistance, and rural or urban residence. We used validated algorithms to define the Charlson comorbidities and hypertension ${ }^{18}$ at baseline using physician claims and data on use of hospitalisation and ambulatory care. The Charlson score was based on the Deyo classification ${ }^{19}$ of the following comorbidities: cerebrovascular disease, peripheral vascular disease, congestive heart failure, cancer, chronic obstructive pulmonary disease, dementia, diabetes with and without complications, AIDS/HIV, metastatic solid tumour, myocardial infarction, mild liver disease, moderate or severe liver disease, paralysis, peptic ulcer disease, and rheumatic disease. We used physician claims and data on use of hospitalisation and ambulatory care to assess for a history of kidney stone at baseline (that is, at least three years before follow-up) using the ICD codes above.

\section{Clinical outcomes}

The primary outcome was ESRD, defined as receipt of a kidney transplant or initiation of chronic dialysis during follow-up, and ascertained by data linkage to the Northern and Southern Alberta Renal Programs. Using a subset of patients with data for serum creatinine concentration, we followed patients from their first available outpatient serum creatinine measurement (May 2002 or later) until March 2009. Patients with estimated glomerular filtration rate $<45 \mathrm{~mL} / \mathrm{min} / 1.73 \mathrm{~m}^{2}$ at baseline were excluded from these analyses. Kidney stones occurring on or before the index date were counted as a prior kidney stone, and only events occurring after the first kidney stone episode were included in the analysis. Outcomes assessed in this subset of patients included new stage $3 \mathrm{~b}-5$ chronic kidney disease (estimated glomerular filtration rate $<45 \mathrm{~mL} / \mathrm{min} / 1.73 \mathrm{~m}^{2}$ ) and sustained doubling of serum creatinine concentration (a surrogate measure for progressive kidney disease). Doubling of serum creatinine was considered to have occurred at the time when a serum creatinine value was found to be twice as high as the first measurement during the study provided that all subsequent values during follow-up remained at least twice as high as at baseline. ${ }^{20}$ Glomerular filtration rate was estimated with the Chronic Kidney Disease Epidemiology Collaboration equation. ${ }^{21}$

\section{Statistical analyses}

We did analyses with Stata/MP 11.2 (www.stata.com) and reported baseline descriptive statistics as counts and percentages or as medians and interquartile ranges, as appropriate. Absolute rates were calculated by totalling the number of events and dividing by the total follow-up time. These are presented as events per 1000000 person days.

Using each participant as the unit of analysis, we estimated the associations between first episode of kidney stone and outcomes by using multivariable Cox proportional hazards models adjusted for all variables presented in table $1 \Downarrow$. For new stage $3 b-5$ chronic kidney disease and sustained doubling of serum creatinine outcomes, we additionally adjusted for baseline estimated glomerular filtration rate. Time of origin was arbitrary (April 1997 or later, as described above in "Population and data sources").

Kidney stone episodes were used to update exposure status during follow-up - meaning that a person experiencing a single stone episode during the course of follow-up would contribute person time to the "no stone" hazard before the stone episode (regardless of prior stone history) and person time to the "stone" hazard after the stone episode. We explored the associations between the outcomes and multiple episodes of stones in further analyses. Tests for trend were performed by assigning 0,1 , or 2 for none, one, or multiple stone episodes, respectively.

We censored follow-up when a participant died, moved out of the province, or reached the end of the study (31 March 2009). We determined that the proportional hazard assumption was satisfied by examining plots of the log-negative log of within-group survivorship probabilities versus log time. The threshold probability for statistical significance was set at 0.05 . Age and sex were explored as potential modifiers of the association between kidney stones and clinical outcomes.

\section{Results}

\section{Characteristics of study patients}

There were 3089194 eligible patients without ESRD (or estimated glomerular filtration rate $<15 \mathrm{~mL} / \mathrm{min} / 1.73 \mathrm{~m}^{2}$ ) at baseline (fig $1 \Downarrow$ ). Of these, 1954836 had outpatient serum creatinine measurements and were included in analyses of new stage $3 b-5$ chronic kidney disease and sustained doubling of serum creatinine level from baseline. The characteristics of the study patients with and without kidney stone episodes are shown in table $1 \Downarrow$. Patients with kidney stones were older and more likely to be men, to have had hypertension, and to have a history of kidney stones.

During the median follow-up period of 11 years (range 1 day to 12 years), $5333(0.2 \%)$ registry patients developed ESRD, 200790 (7\%) died, and 229556 (7\%) moved outside Alberta. In the subset of patients with serum creatinine data, the median 
follow-up period was four years (range 1 day to 7 years), and in this period 68525 (4\%) patients developed stage $3 \mathrm{~b}-5$ chronic kidney disease, $6581(0.3 \%)$ experienced sustained doubling of serum creatinine from baseline, 79787 (4\%) died, and 74843 (4\%) moved outside Alberta.

\section{Relation between kidney stone episodes and ESRD, stage 3b-5 chronic kidney disease, or doubling of serum creatinine concentration}

The adjusted risk of incident ESRD, our primary outcome, was significantly higher in patients with one or more episodes of kidney stones (hazard ratio 2.16 (95\% confidence interval 1.79 to 2.62 ), table $2 \Downarrow$, fig $2 \Downarrow$ ). Results were similar in analyses that did not adjust for prior kidney stones (hazard ratio 2.11 (1.77 to 2.52)) and in analyses that excluded participants with prior kidney stones (hazard ratio 2.06 (1.67 to 2.55)). A significant association between one or more episodes of kidney stones was also observed for the adjusted risk of new stage $3 \mathrm{~b}-5$ chronic kidney disease (hazard ratio 1.74 (1.61 to 1.88)) and sustained doubling of serum creatinine concentration from baseline (1.94 (1.56 to 2.43$)$ )

The absolute increase in the rate of adverse renal outcomes associated with stones was small: the unadjusted rate of ESRD was 2.48 per million person days in people with one or more episodes of stones, compared with 0.52 per million person days in people without stones. Corresponding unadjusted rates of new stage $3 \mathrm{~b}-5$ chronic kidney disease and sustained doubling of serum creatinine from baseline were 61.47 versus 25.53 and 7.69 versus 2.40 respectively.

The risk of adverse renal outcomes associated with kidney stones seemed to increase with the number of stone episodes, since the adjusted risk of ESRD and doubling of serum creatinine were both greater in patients with multiple episodes of stones as compared with those with a single episode or no stones (table $2 \Downarrow)$.

\section{Effect modification by age and sex}

In analyses that separately evaluated the effect modification of age and sex (table $3 \Downarrow$, fig $2 \Downarrow$ ), the adjusted risk of ESRD associated with one or more episodes of stones (compared with those without stones) seemed greater for women than men (P value for interaction 0.003). Results were similar in the other two adverse renal outcomes, although this interaction was of borderline significance for new stage $3 b-5$ chronic kidney disease $(\mathrm{P}=0.06)$. The risk of incident ESRD associated with one or more stone episodes (versus no stones) was not significantly higher in people aged $<50$ years than in those aged $\geq 50(\mathrm{P}=0.11)$, but the risk of the other two adverse renal outcomes was significantly greater in those aged $<50$ than in those who were older (both $\mathrm{P} \leq 0.01$, table $3 \Downarrow$ ). Although the magnitude of the association between stones and adverse outcomes was greater for women than for men and for those aged $<50$ years than for those aged $\geq 50$, the risk of all three outcomes in those with at least one episode of stones was significantly higher than in those without stones in both sexes and both age strata (table $3 \Downarrow$ ). The absolute increase in the rate of ESRD was small for all age and sex subgroups (fig $2 \Downarrow$ ).

Results were similar in analyses that examined the risk of ESRD associated with kidney stones in strata defined by the intersection of age and sex (fig $2 \Downarrow$ ). Specifically, the magnitude of the excess risk remained greater in female and younger participants, and the risk of all three adverse renal outcomes (data not shown) was significantly increased in the four mutually exclusive groups formed by the intersection of age and sex.

\section{Discussion}

Kidney stones are a common and potentially preventable cause of morbidity in the general population. Using a cohort of more than three million people treated in a universal healthcare system, we found that the risks of end stage renal disease (ESRD) and of two other clinically relevant adverse renal outcomes were all significantly higher in people with at least one symptomatic kidney stone episode.

The excess risk of all three adverse renal outcomes that was associated with stone episodes seemed to be greater in women than in men and greater among younger people than in those aged $\geq 50$ years. The observed association between stones and risk was independent of potential confounders (shown in table $1 \Downarrow)$ and seemed to be magnified in people with more than one episode of stones compared with those with a single episode. These findings suggest that kidney stones are an important potential contributor to the risk of ESRD and that patients with prior kidney stones should be considered at increased risk for adverse renal outcomes-especially younger women or those with multiple symptomatic episodes.

\section{Comparison with other studies}

Consistent with our data, a recent prospective cohort study of about 1.5 million people in the United Kingdom found that kidney stones were a significant risk for ESRD in women but not men. ${ }^{14}$ The basis for this finding is unclear, but we speculate that, although men are more likely to develop stones, they are protected from obstructive complications due to differing anatomy. The lack of a significant association between stones and ESRD in men in the UK study ${ }^{14}$ may have been due to the smaller sample size and shorter period of follow-up. In our study, the risk of adverse renal outcomes associated with a kidney stone episode was more pronounced in younger participants-perhaps because of the competing risk of death (or the increased frequency of other risk factors for progressive kidney function loss) in those who were older.

Consistent with our findings, Stankus et al found that a self reported history of kidney stones was associated with a twofold increased risk of ESRD among African-American patients. ${ }^{11}$ Another study examining the impact of kidney stones on renal outcomes found that kidney stones were associated with the development of chronic kidney disease, ${ }^{12}$ but it failed to establish an association between kidney stones and ESRD—perhaps because of its smaller sample size.

\section{Potential mechanisms for the findings}

What mechanisms link kidney stones to adverse renal outcomes? People with rare monogenetic disorders that cause kidney stones (such as primary hyperoxaluria and cystinuria) are at increased risk for ESRD, apparently because of the renal damage caused by stone formation. ${ }^{5-7} 9$ In the absence of such unusual conditions, and given the heterogeneous nature of kidney stones and their formation, the link between stones and adverse renal outcomes is likely multifactorial. ${ }^{22}$ The association between calcium kidney stones and progressive loss of kidney function may be the direct result of progressive calcification within the renal interstitium, and specifically at the tubular basement membrane and around the ducts of Bellini. ${ }^{22}{ }^{23}$ Extension of such calcification into the tubular lumen might cause more renal damage, with potential for progressive scarring, chronic kidney disease, and ultimately ESRD. Alternatively, crystallisation within the tubular lumen itself may cause damage to the tubular epithelium, and/or obstruction leading to progressive scarring. ${ }^{1322}{ }^{23}$ It is also possible that repeated episodes of urinary 
tract obstruction associated with stones could contribute to progressive loss of renal function. Finally, it is possible that surgical or percutaneous treatment of stones (rather than the stones themselves) accounts for the excess risk of kidney function loss. However, since our data do not permit us to address the mechanism linking kidney stones to adverse renal outcomes, these speculations require confirmation in future mechanistic studies.

\section{Limitations}

Our study is limited by the fact that people with kidney stones were identified by their presentation to health services, meaning that our findings do not apply to those who did not seek medical care for a stone episode. Consequently we cannot comment on the association between asymptomatic kidney stones and adverse renal outcomes. Similarly, although we attempted to capture discrete episodes of kidney stones, the algorithm we used requires some assumptions, and some patients were therefore probably misclassified with respect to their number of presentations. Since we prospectively used claims and hospitalisation data to assess the incidence of kidney stones (rather than a history obtained at the time of dialysis initiation), ascertainment bias due to more complete assessment of stones among patients with ESRD is unlikely to have influenced our findings. We were not able to determine the composition of the kidney stones and thus cannot assess the specific risk associated with different stone types. Although we adjusted for numerous characteristics that differ by whether a participant had a kidney stone episode (such as age, prior kidney stone, and other comorbidity), the possibility for residual confounding remains. Patients with stones may be more likely to have serum creatinine concentration measured - and, therefore, we might be more likely to detect a sustained doubling of serum creatinine or new chronic kidney disease in this group. However, given that ESRD is unlikely to remain undetected for long, the primary outcome is unlikely to have been biased in this way. Finally, since the dataset we used did not include genetic information, we cannot assess how many of the patients in our study had a monogenic disorder predisposing to stones. However, given the number of ESRD events in our study, it seems unlikely that our findings are driven by such rare conditions.

\section{Conclusions}

In conclusion, we found a graded association between episodes of kidney stones and the risk of adverse renal outcomes, including ESRD. Further research should be aimed at determining the mechanisms explaining this association and assessing the optimal way to prevent kidney stones in the general population, especially young women.

Contributors: RTA conceived the study. RTA, NW, AB, and MT designed the analysis plan. NW performed the statistical analyses. RTA, NW, and MT wrote the first draft of the study. All authors contributed to the design, interpretation of results, and critical revision of the article for intellectually important content.

Funding: This work was supported by a team grant to the Interdisciplinary Chronic Disease Collaboration from the Alberta Heritage Foundation for Medical Research (AHFMR), the Kidney Foundation of Canada, and by the University Hospital Foundation. RTA is supported by a Clinician-Scientist award from the Canadian Institute of Health Research, a KRESCENT New Investigator award, and an Alberta Innovates Health Solutions Clinical Investigator Award. SS is supported by a KRESCENT
New Investigator award and a Canadian Child Health Clinician Scientist Program Career Development award. GCC is supported by grant DK070756 from the National Institutes of Health. MT is supported by an AHFMR Population Health Scholar award and a Government of Canada Research Chair in the optimal care of people with chronic kidney disease.

Competing interests: All authors have completed the ICMJE uniform disclosure form at ww.icmje.org/coi_disclosure.pdf (available on request from the corresponding author) and declare: no financial relationships with any organisations that might have an interest in the submitted work in the previous three years; and no other relationships or activities that could appear to have influenced the submitted work.

Ethical approval: Our protocol was approved by the University of Alberta Health Research Ethics Board and the University of Calgary Conjoint Health Research Ethics Board.

Data sharing: No additional data available.

1 Boddana P, Caskey F, Casula A, Ansell D. UK Renal Registry 11th annual report (December 2008). Chapter 14: UK Renal Registry and international comparisons. Nephron Clin Pract 2009;111(suppl 1):c269-76.

2 USRDS United States Renal Data System. Incidence and prevalence of ESRD. Am J Kidney Dis 1997;30:S40-53.

3 Stamatelou KK, Francis ME, Jones CA, Nyberg LM, Curhan GC. Time trends in reported prevalence of kidney stones in the United States: 1976-1994. Kidney Int 2003;63:1817-23. Assimos DG, Leslie SW, Ng C, Streem SB, Hart LJ. The impact of cystinuria on renal function. J Urol 2002;168:27-30.

5 Jungers $\mathrm{P}$, Joly D, Barbey F, Choukroun G, Daudon M. ESRD caused by nephrolithiasis: prevalence, mechanisms, and prevention. Am J Kidney Dis 2004:44:799-805

6 Lieske JC, Monico CG, Holmes WS, Bergstralh EJ, Slezak JM, Rohlinger AL, et al. International registry for primary hyperoxaluria. Am J Nephrol 2005;25:290-6.

7 Edvardsson V, Palsson R, Olafsson I, Hjaltadottir G, Laxdal T. Clinical features and genotype of adenine phosphoribosyltransferase deficiency in Iceland. Am J Kidney Dis 2001;38:473-80.

8 Ludwig M, Utsch B, Monnens LA. Recent advances in understanding the clinical and genetic heterogeneity of Dent's disease. Nephrol Dial Transplant 2006;21:2708-17.

9 Tosetto E, Ghiggeri GM, Emma F, Barbano G, Carrea A, Vezzoli G, et al. Phenotypic and genetic heterogeneity in Dent's disease-the results of an Italian collaborative study. Nephrol Dial Transplant 2006;21:2452-63.

10 Collins AJ, Foley RN, Herzog C, Chavers BM, Gilbertson D, Ishani A, et al. Excerpts from the US Renal Data System 2009 annual data report. Am J Kidney Dis 2010;55:S1-420, A6-7.

11 Stankus N, Hammes M, Gillen D, Worcester E. African American ESRD patients have a high pre-dialysis prevalence of kidney stones compared to NHANES III. Urol Res 2007;35:83-7.

12 Rule AD, Bergstralh EJ, Melton LJ 3rd, Li X, Weaver AL, Lieske JC. Kidney stones and the risk for chronic kidney disease. Clin J Am Soc Nephrol 2009:4:804-11.

13 Rule AD, Krambeck AE, Lieske JC. Chronic kidney disease in kidney stone formers. Clin J Am Soc Nephrol 2011;6:2069-75.

14 Hippisley-Cox J, Coupland C. Predicting the risk of chronic kidney disease in men and women in England and Wales: prospective derivation and external validation of the QKidney scores. BMC Fam Pract 2010;11:49.

15 Hemmelgarn BR, Clement F, Manns BJ, Klarenbach S, James MT, Ravani P, et al. Overview of the Alberta Kidney Disease Network. BMC Nephrol 2009;10:30.

16 Sutherland JW, Parks JH, Coe FL. Recurrence after a single renal stone in a community practice. Miner Electrolyte Metab 1985;11:267-9.

17 Trinchieri A, Ostini F, Nespoli R, Rovera F, Montanari E, Zanetti G. A prospective study of recurrence rate and risk factors for recurrence after a first renal stone. J Urol 1999;162:27-30.

18 Quan H, Khan N, Hemmelgarn BR, Tu K, Chen G, Campbell N, et al. Validation of a case definition to define hypertension using administrative data. Hypertension 2009;54:1423-8.

19 Quan H, Sundararajan V, Halfon P, Fong A, Burnand B, Luthi JC, et al. Coding algorithms for defining comorbidities in ICD-9-CM and ICD-10 administrative data. Med Care 2005;43:1130-9.

20 James MT, Hemmelgarn BR, Wiebe N, Pannu N, Manns BJ, Klarenbach SW, et al. Glomerular filtration rate, proteinuria, and the incidence and consequences of acute kidney injury: a cohort study. Lancet 2010;376:2096-103.

21 Levin A, Hemmelgarn B, Culleton B, Tobe S, McFarlane P, Ruzicka M, et al. Guidelines for the management of chronic kidney disease. CMAJ 2008;179:1154-62.

22 Coe FL, Evan AP, Worcester EM, Lingeman JE. Three pathways for human kidney stone formation. Urol Res 2010;38:147-60.

23 Evan AP. Physiopathology and etiology of stone formation in the kidney and the urinary tract. Pediatr Nephrol 2010;25:831-41.

Accepted: 25 July 2012

\section{Cite this as: BMJ 2012;345:e5287}

This is an open-access article distributed under the terms of the Creative Commons Attribution Non-commercial License, which permits use, distribution, and reproduction in any medium, provided the original work is properly cited, the use is non commercial and is otherwise in compliance with the license. See: http://creativecommons.org/licenses/by$\mathrm{nc} / 2.0 /$ and http://creativecommons.org/licenses/by-nc/2.0/legalcode. 


\section{What is already known on this topic}

The association between kidney stones and the risk of subsequent kidney failure is uncertain

\section{What this study adds}

This population based study found that one or more episodes of kidney stones during follow-up increases the risk of developing kidney failure over a median period of 11 years

\section{Tables}

Table 1| Demographic and clinical characteristics of study patients with and without kidney stone episodes. Values are numbers (percentages) of patients unless stated otherwise

\begin{tabular}{|c|c|c|c|c|}
\hline \multirow[b]{2}{*}{ Characteristic } & \multicolumn{2}{|c|}{ Registry dataset* } & \multicolumn{2}{|c|}{ Laboratory dataset $†$} \\
\hline & $\begin{array}{c}\text { Stone }(s) \\
(n=23706)\end{array}$ & $\begin{array}{c}\text { No stone } \\
(n=3 \quad 065 \text { 488) }\end{array}$ & $\begin{array}{c}\text { Stone(s) } \\
(n=11609)\end{array}$ & $\begin{array}{c}\text { No stone } \\
(n=1943 \quad 227)\end{array}$ \\
\hline Median (IQR) age (years) & $46(35.1-60.1)$ & $35.4(23.2-48.6)$ & $51.6(41.2-63.2)$ & $45.9(34-57.6)$ \\
\hline Male & $15686(66.2)$ & $1537388(50.2)$ & $7517(64.8)$ & $869635(44.8)$ \\
\hline Aboriginal & $450(1.9)$ & $84244(2.7)$ & $222(1.9)$ & $47562(2.4)$ \\
\hline Receiving social assistance & $819(3.5)$ & $81852(2.7)$ & $440(3.8)$ & $58305(3)$ \\
\hline Rural residence & $3357(14.3)$ & $389597(12.8)$ & $1788(15.4)$ & $249150(12.8)$ \\
\hline \multicolumn{5}{|l|}{ Comorbidities: } \\
\hline $\begin{array}{l}\text { Median (IQR) Charlson } \\
\text { score } \neq\end{array}$ & $0(0-1)$ & $0(0-0)$ & $0(0-1)$ & $0(0-1)$ \\
\hline Hypertension & $3766(15.9)$ & 252467 (8.2) & $3573(30.8)$ & $394661(20.3)$ \\
\hline Prior nephrolithiasis§ & $4056(17.1)$ & $4150(.1)$ & $5796(49.9)$ & $11216(0.6)$ \\
\hline
\end{tabular}

IQR=interquartile range. CKD chronic kidney disease

*Full set of 3089194 eligible patients without end stage renal disease at baseline.

†Subset of 1954836 patients with serum creatinine measurements available.

$\ddagger$ Charlson score includes AIDS/HIV, metastatic cancer, non-metastatic cancer, cerebral vascular disease, chronic obstructive pulmonary disease, dementia, diabetes, heart failure, mild liver disease, moderate/severe liver disease, myocardial infarction, paraplegia, peptic ulcer, peripheral vascular disease, and rheumatological disease.

$\S N$ Nephrolithiasis occurring within 3 years or 8 years before follow-up period in the registry and laboratory dataset respectively. 
Table 2/ Presence of kidney stones ${ }^{\star}$ and risk of adverse renal outcomes (end stage renal disease, chronic kidney disease (stage $3 \mathrm{~b}-5$ ), and doubling of serum creatinine concentration). Values are multivariable adjusted hazard ratios $\uparrow$ ( $95 \%$ confidence intervals) unless stated otherwise

\begin{tabular}{|c|c|c|c|}
\hline & End stage renal disease & Chronic kidney disease & Doubled serum creatinine \\
\hline No of events/No of patients (\%) & $5306 / 3068816(0.2)$ & 68 453/1 $952198(3.5)$ & 6574/1 $952198(0.3)$ \\
\hline $\begin{array}{l}\text { First kidney stone episode during follow-up } \\
\text { ( } v \text { no stone) }\end{array}$ & 2.16 (1.79 to 2.62$)$ & $1.74(1.61$ to 1.88$)$ & 1.94 (1.56 to 2.43$)$ \\
\hline No stones & 1.00 & 1.00 & 1.00 \\
\hline $\begin{array}{l}\text { Single kidney stone episode during follow-up } \\
\text { ( } v \text { no stone) }\end{array}$ & 2.11 (1.69 to 2.63$)$ & $1.73(1.58$ to 1.88$)$ & $1.84(1.43$ to 2.36$)$ \\
\hline $\begin{array}{l}\geq 2 \text { kidney stone episodes during follow-up } \\
\text { ( } v \text { no stone) }\end{array}$ & 2.31 (1.66 to 3.21$)$ & $1.63(1.37$ to 1.95$)$ & $2.22(1.49$ to 3.33$)$ \\
\hline $\mathrm{P}$ value for trend (No of stone episodes) & $<0.001$ & $<0.001$ & $<0.001$ \\
\hline
\end{tabular}

"Kidney stones were treated as a time-varying exposure: all participants were classified as "unexposed" at the start of follow-up, but, once a kidney stone occurred, participants were classified as "exposed" for the remainder of the study.

†Results were adjusted for age (18-49.9, 50-69.9, $\geq 70$ years), sex, Aboriginal, receipt of social assistance, rural residence, comorbidities (Charlson score and hypertension), and prior nephrolithiasis. New stage $3 b-5$ chronic kidney disease and doubling of serum creatinine were evaluated in a subset of 1954836 patients and also adjusted for baseline estimated glomerular filtration rate. The referent group for all analyses was those who had no stone during follow-up. 
Table 3| Presence of kidney stones* and risk of adverse renal outcomes (end stage renal disease, chronic kidney disease (stage $3 \mathrm{~b}-5$ ), and doubling of serum creatinine concentration) with one-way stratification on age and sex. Values are multivariable adjusted hazard ratios $\uparrow(95 \%$ confidence intervals) unless stated otherwise

\begin{tabular}{|c|c|c|c|}
\hline & End stage renal disease & Chronic kidney disease & Doubled serum creatinine \\
\hline \multicolumn{4}{|l|}{ Age } \\
\hline \multicolumn{4}{|l|}{$\geq 50$ years: } \\
\hline No of events/No of patients (\%) & $3554 / 722035(0.5)$ & 64 471/794 $709(8.1)$ & $5443 / 795087(0.7)$ \\
\hline $\begin{array}{l}\text { First kidney stone episode during follow-up ( } v \\
\text { no stone) }\end{array}$ & 2.01 (1.61 to 2.49 ) & $1.63(1.50$ to 1.77$)$ & $1.77(1.38$ to 2.25$)$ \\
\hline \multicolumn{4}{|l|}{$<50$ years: } \\
\hline No of events/No of patients (\%) & $1762 / 2370325(0.07)$ & $3982 / 1169088(0.3)$ & $1131 / 1169121(0.1)$ \\
\hline $\begin{array}{l}\text { First kidney stone episode during follow-up ( } v \\
\text { no stone) }\end{array}$ & 2.81 (1.96 to 4.03 ) & 3.65 (2.92 to 4.55$)$ & 3.38 (2.14 to 5.34$)$ \\
\hline$P$ value for interaction of age and kidney stones & 0.11 & $<0.001$ & 0.01 \\
\hline \multicolumn{4}{|l|}{ Sex } \\
\hline \multicolumn{4}{|l|}{ Male: } \\
\hline No of events/No of patients (\%) & $3270 / 1558442(0.2)$ & 29 325/883 472 (3.6) & $3275 / 883756(0.4)$ \\
\hline $\begin{array}{l}\text { First kidney stone episode during follow-up ( } v \\
\text { no stone) }\end{array}$ & 1.87 (1.49 to 2.34$)$ & $1.67(1.52$ to 1.83$)$ & $1.70(1.30$ to 2.21$)$ \\
\hline \multicolumn{4}{|l|}{ Female: } \\
\hline No of events/No of patients (\%) & 2036/1 $533918(0.1)$ & $39128 / 1080128(3.6)$ & $3299 / 1080452(0.3)$ \\
\hline $\begin{array}{l}\text { First kidney stone episode during follow-up ( } v \\
\text { no stone) }\end{array}$ & 3.36 (2.42 to 4.66$)$ & 1.94 (1.69 to 2.24$)$ & 2.65 (1.87 to 3.77$)$ \\
\hline$P$ value for interaction of sex and kidney stones & 0.003 & 0.06 & 0.03 \\
\hline
\end{tabular}

"Kidney stones were treated as a time-varying exposure: all participants were classified as "unexposed" at the start of follow-up, but, once a kidney stone occurred, participants were classified as "exposed" for the remainder of the study.

†Results were adjusted for age (18-49.9, 50-69.9, $\geq 70$ years), sex, Aboriginal, receipt of social assistance, rural residence, comorbidities (Charlson score and hypertension), and prior nephrolithiasis. New stage 3b-5 chronic kidney disease and doubling of serum creatinine were evaluated in a subset of 1954836 patients and also adjusted for baseline estimated glomerular filtration rate. The referent group for all analyses was those who had no stone during follow-up. 


\section{Figures}

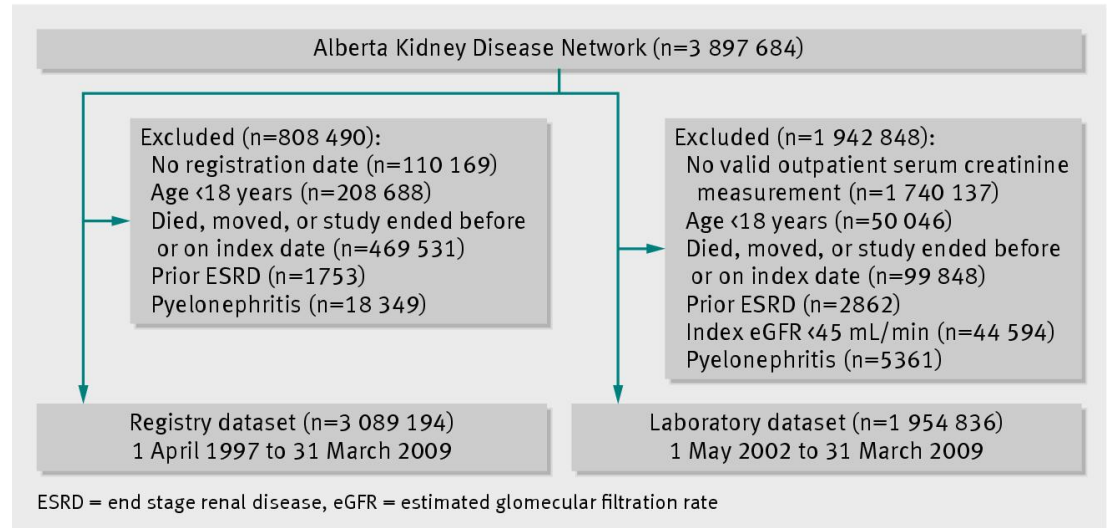

Fig 1 Patient flow through study

\begin{tabular}{|c|c|c|c|c|c|}
\hline \multirow[b]{2}{*}{ Subgroup } & \multicolumn{2}{|c|}{ Unadjusted ESRD rate } & \multirow[b]{2}{*}{$\begin{array}{l}\text { Hazard ratio } \\
(95 \% \mathrm{CI})\end{array}$} & & \multirow[b]{2}{*}{$\begin{array}{l}\text { Hazard ratio } \\
(95 \% \mathrm{Cl})\end{array}$} \\
\hline & $\begin{array}{l}\text { Kidney } \\
\text { stones }\end{array}$ & $\begin{array}{l}\text { No kidney } \\
\text { stones }\end{array}$ & & $\begin{array}{l}\text { atio } \\
\text { a) }\end{array}$ & \\
\hline \multicolumn{6}{|l|}{$\begin{array}{l}\text { First kidney stone episode during } \\
\text { follow-up } v \text { no kidney stones }\end{array}$} \\
\hline Overall & 2.48 & 0.52 & & $\rightarrow-$ & 2.16 (1.79 to 2.62$)$ \\
\hline$\geq 50$ years old & 4.68 & 1.43 & & $\rightarrow$ & 2.01 (1.61 to 2.49 ) \\
\hline$<50$ years old & 1.00 & 0.23 & & $\longrightarrow$ & 2.81 (1.96 to 4.03$)$ \\
\hline Men & 2.65 & 0.64 & & $\rightarrow$ & 1.87 (1.49 to 2.34$)$ \\
\hline Women & 2.16 & 0.40 & & $\longrightarrow-$ & $3.36(2.42$ to 4.66$)$ \\
\hline \multicolumn{6}{|l|}{ Men } \\
\hline$\geq 50$ years old & 4.44 & 1.85 & & $\rightarrow-$ & 1.85 (1.46 to 2.34$)$ \\
\hline$<50$ years old & 1.13 & 0.29 & & $\longrightarrow$ & $2.43(1.60$ to 3.70$)$ \\
\hline \multicolumn{6}{|l|}{ Women } \\
\hline$\geq 50$ years old & 5.44 & 1.06 & & $\longrightarrow$ & 2.72 (1.87 to 3.96$)$ \\
\hline$<50$ years old & 0.81 & 0.17 & & $\longrightarrow$ & 3.58 (2.38 to 5.38$)$ \\
\hline \multicolumn{6}{|c|}{$\begin{array}{l}\text { No of kidney stone episodes during } \\
\text { follow-up } v \text { no kidney stones }\end{array}$} \\
\hline First kidney stone episode & 2.36 & - & & $\rightarrow-$ & 2.11 (1.69 to 2.63 ) \\
\hline \multirow[t]{2}{*}{ Second kidney stone episode } & 2.82 & - & & $\longrightarrow$ & 2.31 (1.66 to 3.21$)$ \\
\hline & & 0.2 & 0.5 & 2 & \\
\hline
\end{tabular}

Fig 2 Forest plot of multivariable adjusted hazard ratios for kidney stones and ESRD. Absolute rates are ESRD rates per 1000000 person days 